\title{
A coupled HDG-FV method for unsteady compressible flows
}

\author{
Sanjay Komala-Sheshachala, Ruben Sevilla* and Oubay Hassan \\ Zienkiewicz Centre for Computational Engineering, \\ Swansea University, Swansea, SA1 8EN, Wales, UK \\ e-mail: r.sevilla@swansea.ac.uk
}

\begin{abstract}
Second-order vertex-centred finite volume (FV) methods are still the predominant technique used in commercial and industrial computational fluid dynamics solvers. For steady problems, low-order mesh generation and FV methods have achieved a level of maturity that enables their routine application for problems of industrial interest involving complex geometries.

For simulating transient phenomena (e.g. manoeuvring, gust), low order methods are known to introduce significant dissipation and dispersion errors. In such situations, a new refined mesh for every transient simulation has to be regenerated.

In the two last decades, high-order methods have attracted significant attention due to the possibility to decrease the number of degrees of freedom required to reach a prescribed level of accuracy, when compared to low order methods. In some studies, the reduction in terms of computing time has also been demonstrated [1]. Out of the many possible high-order methods available, the hybridisable discontinuous Galerkin (HDG) $[2,3]$ is an attractive option due to the reduced number of degrees of freedom, compared to other DG methods, and the ability to introduce the stabilisation required in convection dominated flows [4].

One of the areas hampering the application of high-order methods to industrially relevant problems is the need for robust curvilinear mesh generators, capable of producing high quality meshes for complex geometries [5]. Despite some approaches are recently available [6], it seems that the robustness of these techniques has not yet reached the level required by industry.

This work proposes a strong coupling of the vertex-centred FV method and the HDG method for simulating unsteady flows on meshes designed for steady simulations [7]. Finite volumes are employed in the vicinity of the aerodynamic shapes, where the mesh, initially designed for steady, is able to provide the required accuracy. In the rest of the domain, where the mesh is too coarse to capture the transient effects, the high-order HDG approach is employed. To ensure a good representation of the solution in coarse elements, the degree of the approximation is adapted.

The proposed technique is first tested using two and three dimensional problems with analytical solution, showing the optimal convergence properties of the proposed scheme. Its application to problems involving rarefaction waves, contact discontinuities and shocks is also presented. This example shows that the quality of the approximation is not dependent on the relative position of the discontinuities and the interface between the FV and the HDG methods. Finally, the method is applied to two and three dimensional problems involving the simulation of a time-harmonic gust impinging on a wing and on a wing-tail configuration are presented.

As an example, figure 1 shows the results of a gust simulation with a wing-tail configuration immersed in an inviscid transonic flow at free stream Mach number $M_{\infty}=0.8$. An unstructured triangular mesh with 12,504 elements was generated, with localised mesh refinement around the wing and tail. This mesh, suitable for a steady state simulation is then partitioned in two regions. In the region where the elements are small enough to capture the gust perturbation, a standard FV scheme is employed whereas in the rest of the domain an HDG approach is used. In the HDG region, the degree of the approximation is adapted.
\end{abstract}




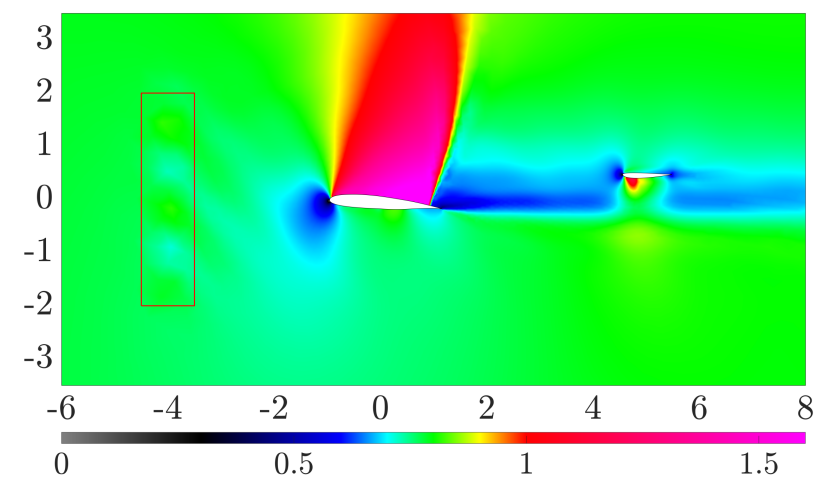

(a) FV

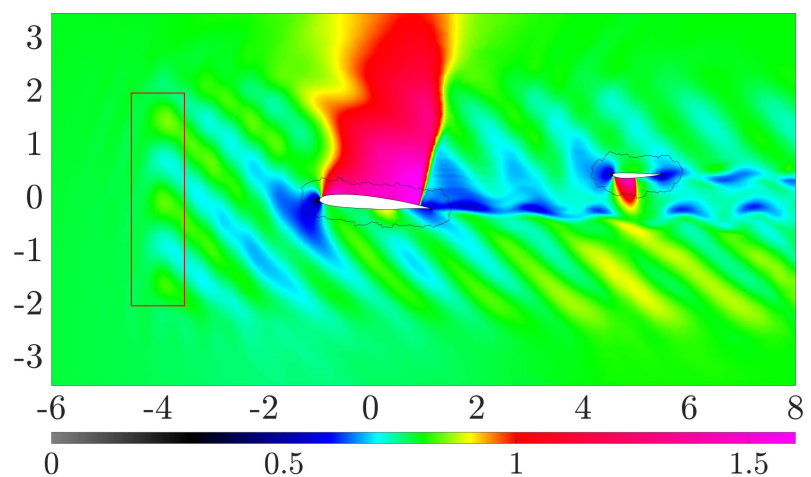

(b) HDG-FV

Figure 1: Mach number distribution for the simulation of the wind gust impinging in a wing-tail configuration after the time harmonic steady state is reached.

The results illustrate the substantial dissipation introduced by the FV scheme when the coarse mesh, suitable for a steady state simulation, is used. Instead, the solution with the proposed HDGscheme is able to capture the perturbation of the velocity induced by the gust, not only impinging in the aerofoil, but also arriving to the tail and interacting with the strong shocks on both the aerofoil and the tail.

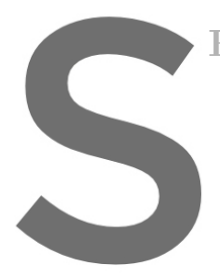

REFERENCES

[1] R. Sevilla, O. Ha Mechanics and Engi
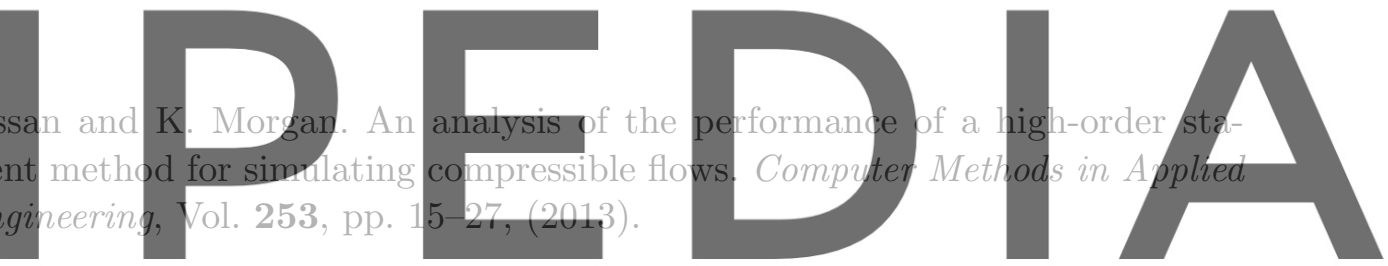

[2] B. Cockburn, J. Gopalakrishnan and R. Lazarov. Unified hybridization of discontinuous

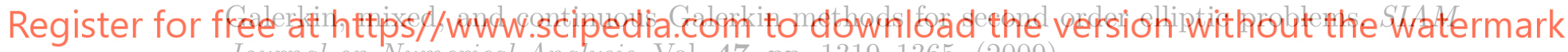
Journal on Numerical Analysis, Vol. 47, pp. 1319-1365, (2009).

[3] Mi. Giacomini, R. Sevilia and A Huerta. HDGiab: An open-source implementation of the hybridisable discontinuous Galerkin method in MATLAB. Archives of Computational Methods in Engineering, pp. 1-46, 2020.

[4] J. Vila-Pérez, M. Giacomini, R. Sevilla and A Huerta. Hybridisable discontinuous Galerkin formulation of compressible flows. Archives of Computational Methods in Engineering, pp. 1-32, 2020.

[5] R. Sevilla, S. Fernández-Méndez and A Huerta. NURBS-enhanced finite element method (NEFEM): A seamless bridge between CAD and FEM. Archives of Computational Methods in Engineering, Vol. 18, pp. 441-484, (2011).

[6] Z.Q. Xie, R. Sevilla, O. Hassan and K. Morgan. The generation of arbitrary order curved meshes for 3D finite element analysis. Computational Mechanics, Vol. 51, pp. 361-374, (2013).

[7] S. Komala-Sheshachala, R. Sevilla and O Hassan. A coupled HDG-FV scheme for the simulation of transient inviscid compressible flows. Computers $\&$ Fluids, 104495, (2020). 